\title{
Keynote Lecture: Sustainable and increasingly efficient road transport for the next generations - continuous improvement through an integrated approach
}

Ron Borsboom, DAF Trucks NV, Netherlands

Zum Zeitpunkt der Drucklegung lagen diese Unterlagen noch nicht vor. Wir bitten um Verständnis. 